\title{
Pathophysiological Contribution of Vascular Function to Baroreflex Regulation in Hypertension
}

\author{
Hirofumi Tomiyama, MD; Chisa Matsumoto, MD; Kazutaka Kimura, MD; \\ Mari Odaira, MD; Kazuki Shiina, MD; Akira Yamashina, MD
}

\begin{abstract}
Background: We examined which pathophysiological abnormalities of vascular function might be closely associated with abnormal baroreflex regulation in subjects with hypertension.

Methods and Results: In the cross-sectional assessment, 280 subjects with hypertension were enrolled for measurement of brachial-ankle pulse wave velocity (baPWV), radial augmentation index (rAl), flow-mediated vasodilatation (FMD) of the brachial artery and baroreceptor sensitivity (BRS). These parameters were measured again as prospective assessment in some of these subjects. In the cross-sectional assessment, after adjustment for confounding variables including anti-hypertensive medication, the baPWV, but not the rAI or FMD, was found to have a significant independent relationship with BRS (standardization coefficient, $-0.149, \mathrm{P}<0.043$ ). In the subjects who were newly started on anti-hypertensive medication $(n=40)$, regression of baPWV before and 1 year after the start of medication was significantly associated with change in BRS during the same period. In subjects already on anti-hypertensive medication $(n=92)$ also, the evolutional change of baPWV over a follow-up period $>1.5$ years was significantly associated with change in BRS during the same period.
\end{abstract}

Conclusions: Increased stiffness of the large- to middle-sized arteries, rather than abnormal central hemodynamics or endothelial dysfunction, appears to contribute to abnormal baroreflex regulation in patients with hypertension. (Circ J 2014; 78: 1414-1419)

Key Words: Arterial stiffness; Baroreceptor sensitivity; Central hemodynamics; Endothelial function

$\mathbf{P}$ ulse wave velocity (PWV), augmentation index (AI)/ central blood pressure (CBP), and/or flow-mediated vasodilatation (FMD) of the brachial artery are wellknown markers of vascular function, 1,2 and have been reported to predict risk of development of cardiovascular events in subjects with hypertension. ${ }^{3-5}$ The mechanisms underlying the involvement of unfavorable pathophysiological conditions in the development of cardiovascular disease, however, have not yet been clarified in detail.

Considering the several findings described here, it is possible that abnormal autonomic nervous functions may be an important factor contributing to the risk of cardiovascular disease associated with abnormal vascular function. ${ }^{6}$ Baroreceptor sensitivity (BRS) is one of the components of the autonomic nervous system involved in cardiovascular regulation, ${ }^{7,8}$ and several studies have noted impaired BRS in subjects with hypertension. ${ }^{9,10}$ Barosensory vessel stretch and neural transduction of this stretch into the vagal outflow are key steps in baroreflex regulation. ${ }^{11}$ Some cross-sectional studies have reported that stiffness of the large arteries, as reflected by PWV, which would be expected to be associated with impaired baro- sensory stretch, is negatively associated with BRS. ${ }^{12-16}$ No longitudinal study, however, has examined the association between change in PWV over time with that of BRS during the same period.

Concerning other markers of vascular function, it has been reported that central hemodynamics, as estimated by $\mathrm{AI}$, rather than brachial blood pressure, exert an influence on baroreflex regulation. ${ }^{9}, 10$ In contrast, FMD reflects the endothelial nitric oxide (NO) bio-availability, and NO directly contributes to baroreflex regulation in the brain, besides also contributing indirectly via its effect on arterial elasticity, which is a factor influencing barosensory vessel stretch. ${ }^{17,18}$ Furthermore, endothelial function-related substances such as prostacycline or oxygen free radicals may also directly modulate BRS. ${ }^{19,20}$ The significance of the association of AI/CBP or FMD with BRS, however, has not been fully clarified.

Confirmation of the association between longitudinal change in markers of vascular function and that in BRS may support the idea that pathophysiological abnormalities of vascular function contribute to abnormal baroreflex regulation. Therefore, the present study was conducted to examine which markers of

Received January 29, 2014; revised manuscript received February 26, 2014; accepted February 27, 2014; released online April 3, 2014

Time for primary review: 18 days

Second Department of Internal Medicine, Tokyo Medical University, Tokyo, Japan

Mailing address: Hirofumi Tomiyama, MD, Second Department of Internal Medicine, Tokyo Medical University, 6-7-1 Nishi-Shinjuku,

Tokyo 160-0023, Japan. E-mail: tomiyama@tokyo-med.ac.jp

ISSN-1346-9843 doi:10.1253/circj.CJ-14-0064

All rights are reserved to the Japanese Circulation Society. For permissions, please e-mail: cj@j-circ.or.jp 
vascular function (ie, PWV, AI/CBP and FMD) have a close association with BRS on cross-sectional assessment and longitudinal assessments (evolutional change and regression) in subjects with hypertension.

\section{Methods}

\section{Subjects}

This study was conducted at the outpatient clinic of Tokyo Medical University Hospital from July 2007 to December 2012. Among the consecutive patients with hypertension who visited the outpatient clinic of Tokyo Medical University Hospital for the management of hypertension, subjects who were scheduled to start on anti-hypertensive medication according to the Japanese Society of Hypertension (JSH) guidelines and subjects who were already on anti-hypertensive medication for at least the previous 6 months were asked to participate in this study (measurement of vascular function and assessment of BRS). Written informed consent was obtained from all subjects prior to participation in the study. The protocol of the study was approved by the Ethics Committee of Tokyo Medical University.

Subjects on $\beta$-blockers and/or with serious underlying medical problems (eg, cardiovascular disease such as coronary artery disease, heart failure, peripheral arterial disease or stroke, other diseases requiring specific medical treatment such as steroid or anti-inflammatory drug therapy) were excluded.

\section{Study Design}

Cross-Sectional Assessment In addition to routine investigations (blood chemistry and electrocardiogram) for the management of hypertension, vascular function tests (ie, brachialankle PWV [baPWV], radial AI [rAI], and FMD) and assessment of BRS were conducted.

Longitudinal Assessment Among the study subjects who underwent cross-sectional assessment, (1) vascular function tests and assessment of BRS were conducted again 1 year after the first assessment in the subjects who were scheduled to start on anti-hypertensive medication according to the JSH guidelines; and (2) these assessments were conducted at 1.5-year intervals from the first assessment in the subjects who were already on anti-hypertensive medication.

In the management of hypertension, target blood pressure was set at $\leq 140 / 90 \mathrm{mmHg}$, and anti-hypertensive medication was selected by the attending physicians. Therapeutic lifestyle modification was also recommended to all study subjects in accordance with the JSH guidelines.

\section{Measurements}

Vascular function tests (FMD, rAI/CBP and baPWV) and assessment of BRS were conducted on the same day, and rAI/ $\mathrm{CBP}$ and baPWV were measured after measurement of FMD of the brachial artery.

FMD The method of measurement of FMD has been described in detail elsewhere. ${ }^{21}$ The subjects were instructed to fast for at least $6 \mathrm{~h}$, and to abstain from alcohol, smoking, caffeine and antioxidant vitamins for at least $12 \mathrm{~h}$ prior to the measurements. They were asked to rest in the sitting position in a quiet, dark, air-conditioned room $\left(24-26^{\circ} \mathrm{C}\right)$ for $5 \mathrm{~min}$, and asked to rest again for at least $15 \mathrm{~min}$ in the supine position in the same room before the measurement of FMD. Ultrasound measurement was done according to the guidelines for ultrasound assessment of FMD. ${ }^{21}$

Using high-resolution ultrasound with a $10-\mathrm{MHz}$ linear array transducer, longitudinal images of the right brachial artery were recorded at baseline and then continuously for $\geq 3 \mathrm{~min}$ after cuff

\begin{tabular}{lc}
\hline \multicolumn{2}{|c|}{ Table 1. Subject Clinical Characteristics } \\
No. subjects & \\
Age (years) & 280 \\
Gender (M/F) & $60 \pm 10$ \\
BMl (kg/m²) & $194 / 86$ \\
Smokers & $24.4 \pm 3.7$ \\
Brachial SBP (mmHg) & $58(21)$ \\
Brachial DBP (mmHg) & $142 \pm 17$ \\
HR (beats/min) & $86 \pm 14$ \\
Serum LDL-C (mg/dl) & $72 \pm 12$ \\
Serum TG (mg/dl) & $115 \pm 38$ \\
Serum HDL-C (mg/dl) & $136 \pm 98$ \\
FPG (mg/dl) & $59 \pm 15$ \\
Serum Crnn (mg/dl) & $105 \pm 19$ \\
baPWV (cm/s) & $0.76 \pm 0.20$ \\
rAl (\%) & $1636 \pm 282$ \\
SP2 (mmHg) & $79 \pm 15$ \\
FMD (\%) & $130 \pm 20$ \\
BRS (ms/mmHg) & $4.0 \pm 2.4$ \\
Anti-hypertensive medication: no/yes (n) & $7.7 \pm 3.7$ \\
Medication & $62 / 218$ \\
CCB & \\
RA blockers & $149(53)$ \\
Diuretics & $150(54)$ \\
Statin & $64(23)$ \\
Oral medication for DM & $54(19)$ \\
& $33(12)$ \\
\hline
\end{tabular}

Data given as mean \pm SD or $\mathrm{n}(\%)$.

baPWV, brachial-ankle pulse wave velocity; BMI, body mass index; BRS, baroreceptor sensitivity; CCB, calcium channel blocker; Crnn, creatinine; DBP, diastolic blood pressure; DM, diabetes mellitus; FMD, flow-mediated vasodilatation of brachial artery; FPG, fasting plasma glucose; HDL-C, high-density lipoprotein cholesterol; HR, heart rate; LDL-C, low-density lipoprotein cholesterol; RA blocker, angiotensin II receptor blocker or angiotensin-converting enzyme inhibitor; rAl, radial augmentation index; SBP, systolic blood pressure; SP2, second peak of radial pressure wave form; TG, triglycerides.

deflation following suprasystolic compression $(50 \mathrm{mmHg}$ over systolic blood pressure [SBP]) of the right forearm for $5 \mathrm{~min}$. The diastolic diameter of the brachial artery was determined semi-automatically using an instrument equipped with software for monitoring brachial artery diameter (Unex, Nagoya, Japan). A segment with clear anterior (intima-media) and posterior (intima-media) interfaces was manually determined. These border interfaces were identified automatically on the A-mode waves as a signal from the intima-media complex, and changes in the diastolic diameter were continuously recorded.

Al Blood pressure was determined in the right upper arm using the oscillometric method (HEM-907; Omron Healthcare, Kyoto, Japan). Immediately after this measurement, the left radial arterial waveform was recorded using an arterial applanation tonometry probe incorporating an array of 40 micropiezoresistive transducers (HEM-9010AI; Omron Healthcare). ${ }^{17,18}$ Then, the first and second peaks of the peripheral systolic pressure (SP1 and SP2) and peripheral diastolic blood pressure (DBP) were automatically detected using the fourth derivatives for each radial arterial waveform, and averaged. rAI was calculated as follows: $(\mathrm{SP} 2-\mathrm{DBP}) /(\mathrm{SP} 1-\mathrm{DBP}) \times 100(\%){ }^{22}$

baPWV This was measured using a volume-plethysmograph (Form/ABI; Colin, Komaki, Japan) in accordance with a pre- 


\begin{tabular}{|lccrr|}
\hline Table 2. Significant Association With BRS: Univariate Analysis & & & \\
Variables & $\begin{array}{c}\text { Non-standardization } \\
\text { coefficient (SE) }\end{array}$ & $\begin{array}{c}\text { Standardization } \\
\text { coefficient }\end{array}$ & t-value & P-value \\
Age & $-0.058(0.021)$ & -0.165 & -2.782 & 0.006 \\
Gender & $1.431(0.468)$ & 0.180 & 3.059 & 0.002 \\
BMI & $0.052(0.059)$ & 0.053 & 0.884 & 0.377 \\
Smokers & $-0.438(0.541)$ & -0.049 & -0.811 & 0.418 \\
Brachial SBP & $-0.035(0.013)$ & -0.164 & -2.771 & 0.006 \\
HR & $-0.074(0.018)$ & -0.236 & -4.058 & $<0.001$ \\
baPWV & $-0.003(0.001)$ & -0.255 & -4.405 & $<0.001$ \\
rAI & $-0.012(0.015)$ & -0.051 & -0.871 & 0.398 \\
SP2 & $-0.027(0.011)$ & -0.148 & -2.499 & 0.013 \\
FMD & $-0.030(0.091)$ & -0.020 & -0.330 & 0.741 \\
\hline
\end{tabular}

Abbreviations as in Table 1.

\begin{tabular}{|c|c|c|c|c|}
\hline $\begin{array}{l}\text { Significant } \\
\text { variable }\end{array}$ & $\begin{array}{l}\text { Non-standardization } \\
\text { coefficient (SE) }\end{array}$ & $\begin{array}{l}\text { Standardization } \\
\text { coefficient }\end{array}$ & t-value & P-value \\
\hline \multicolumn{5}{|l|}{ Total $R^{2}=0.138$} \\
\hline baPWV & $-0.002(0.001)$ & -0.149 & -2.038 & 0.043 \\
\hline \multicolumn{5}{|l|}{ Total $R^{2}=0.131$} \\
\hline SP2 & $-0.040(0.028)$ & -0.219 & -1.416 & 0.158 \\
\hline
\end{tabular}

Covariates: age, gender, smokers, BMI, brachial SBP, HR, CCB, RA blockers, diuretics, statins, and oral medication for DM.

Abbreviations as in Table 1.

viously described methodology. ${ }^{23}$ In brief, occlusion cuffs, which were connected to both the plethysmographic and oscillometric sensors, were tied around both the upper arms and ankles of the subject lying in the supine position. The brachial and post-tibial arterial pressures were measured using the oscillometric sensor. The measurements were conducted after the subject had rested for at least $5 \mathrm{~min}$ in the supine position.

Reproducibility of Measurement Reproducibility of FMD, rAI and baPWV measurements at Tokyo Medical University Hospital has been described elsewhere. ${ }^{22} 24$

BRS Assessment During the resting period for FMD measurement, assessment of BRS was conducted. SBP and heart rate were recorded for $5 \mathrm{~min}$ in the supine position using arterial tonometry and electrocardiography (Jentow-7700; Omron Colin). These measurements were not controlled for the respiratory phase. Spontaneous BRS was determined by means of spectral analysis from these samples using commercial software (GMview II; Signalysis, Tokyo, Japan). ${ }^{25,26}$ In relation to the assessment of BRS, the correlation coefficient for SBP and R-R interval was $\geq 0.85$ for all patients

Laboratory Measurement Serum high-density lipoprotein cholesterol, low-density lipoprotein cholesterol, triglycerides and creatinine, and fasting plasma glucose were measured enzymatically in fasting blood samples.

\section{Statistical Analysis}

All data are expressed as mean $\pm \mathrm{SD}$. The difference between the start and the end of the follow-up period was calculated as a delta change: values at the end of follow-up minus those at the start of the follow-up period. Relationships between the variables were assessed on univariate linear regression analysis and multivariate linear regression analysis. In multivariate linear regression analysis, covariates for adjustment included antihypertensive medication (ie, calcium channel blockers, angio- tensin II receptor blockers or angiotensin-converting enzyme inhibitors, and diuretics), statins, or oral medication for diabetes mellitus. Differences between the start and end of follow-up were evaluated using paired t-test. $\mathrm{P}<0.05$ was considered to indicate statistically significant difference. All statistical analysis was done using IBM/SPSS (19.0J for Windows, IBM/SPSS, Chicago, IL, USA).

\section{Results}

\section{Cross-Sectional Assessment}

A total of 280 patients were entered into the present study protocol. Table 1 lists subject clinical characteristics; 62 subjects were scheduled to start on anti-hypertensive medication and 218 were already receiving anti-hypertensive medication (except $\beta$-blockers) for at least the previous 6 months. Table 2 summarizes the results of univariate linear regression analysis to assess the significance of the relationships of variables with BRS. Among the markers of vascular function, baPWV and SP2, but not rAI and FMD, had a significant correlation with BRS. After adjustment including anti-hypertensive medication, multivariate linear regression analysis showed that baPWV, but not SP2, had a significant independent association with BRS (Table 3).

\section{Longitudinal Assessment}

Regression Change of Vascular Function Parameters and BRS Among the 62 subjects who were scheduled to start on anti-hypertensive medication, 42 subjects agreed to be followed at the outpatient department of Tokyo Medical University Hospital, and vascular function tests and assessment of BRS were successfully conducted 1 year after the start of the antihypertensive medication ( 2 subjects were excluded due to prescription of $\beta$-blockers). In addition to brachial blood pressure, 


\begin{tabular}{|c|c|c|c|c|c|c|}
\hline \multirow{2}{*}{ Variables } & \multicolumn{3}{|c|}{ Regression change } & \multicolumn{3}{|c|}{ Evolutional change } \\
\hline & Baseline & 1 year & P-value & Baseline & End & P-value \\
\hline No. subjects & 40 & & & 92 & & \\
\hline Age (years) & $55 \pm 10$ & & & $60 \pm 10$ & & \\
\hline Gender (M/F) & $28 / 12$ & & & $64 / 28$ & & \\
\hline BMI $\left(\mathrm{kg} / \mathrm{m}^{2}\right)$ & $23.6 \pm 3.1$ & $23.7 \pm 3.2$ & 0.360 & $24.6 \pm 4.0$ & $24.7 \pm 4.0$ & 0.354 \\
\hline Smokers & $6(16)$ & $4(10)$ & $<0.001$ & $19(21)$ & 17 (19) & $<0.001$ \\
\hline Brachial SBP (mmHg) & $155 \pm 17$ & $137 \pm 12$ & $<0.001$ & $138 \pm 15$ & $141 \pm 12$ & 0.095 \\
\hline Brachial DBP (mmHg) & $98 \pm 15$ & $89 \pm 11$ & $<0.001$ & $83 \pm 12$ & $84 \pm 11$ & 0.123 \\
\hline $\mathrm{HR}$ (beats/min) & $74 \pm 11$ & $69 \pm 12$ & $<0.001$ & $70 \pm 11$ & $66 \pm 9$ & $<0.001$ \\
\hline Serum LDL-C (mg/dl) & $113 \pm 36$ & $115 \pm 41$ & 0.750 & $121 \pm 28$ & $114 \pm 35$ & 0.089 \\
\hline Serum TG (mg/dl) & $123 \pm 76$ & $131 \pm 71$ & 0.430 & $135 \pm 85$ & $133 \pm 73$ & 0.755 \\
\hline Serum HDL-C (mg/dl) & $59 \pm 17$ & $57 \pm 16$ & 0.069 & $58 \pm 15$ & $58 \pm 15$ & 0.696 \\
\hline FPG (mg/dl) & $103 \pm 16$ & $101 \pm 17$ & 0.413 & $105 \pm 18$ & $105 \pm 18$ & 0.751 \\
\hline Serum Crnn (mg/dl) & $0.71 \pm 0.16$ & $0.74 \pm 0.16$ & 0.058 & $0.75 \pm 0.17$ & $0.74 \pm 0.17$ & 0.325 \\
\hline baPWV (cm/s) & $1676 \pm 202$ & $1498 \pm 212$ & $<0.001$ & $1597 \pm 275$ & $1618 \pm 271$ & 0.177 \\
\hline rAl & $80 \pm 13$ & $79 \pm 13$ & 0.638 & $79 \pm 15$ & $85 \pm 12$ & $<0.001$ \\
\hline $\mathrm{SP} 2(\mathrm{mmHg})$ & $144 \pm 19$ & $127 \pm 15$ & $<0.001$ & $126 \pm 18$ & $132 \pm 15$ & $<0.001$ \\
\hline FMD (\%) & $4.7 \pm 2.8$ & $4.4 \pm 2.7$ & 0.194 & $3.8 \pm 2.0$ & $3.9 \pm 2.0$ & 0.777 \\
\hline $\mathrm{BRS}(\mathrm{ms} / \mathrm{mmHg})$ & $6.8 \pm 2.9$ & $9.6 \pm 6.2$ & $<0.001$ & $7.8 \pm 4.0$ & $7.1 \pm 3.6$ & 0.004 \\
\hline \multicolumn{7}{|l|}{ Medication } \\
\hline СCB & & $17(43)$ & & $64(70)$ & $66(72)$ & \\
\hline ARB & & $30(75)$ & & $63(69)$ & $66(72)$ & \\
\hline Diuretic & & $16(40)$ & & $29(32)$ & $33(36)$ & \\
\hline Statin & $5(13)$ & & & $14(15)$ & & \\
\hline Oral medication for DM & $3(8)$ & & & $12(13)$ & & \\
\hline
\end{tabular}

Data given as mean $\pm \mathrm{SD}$ or $\mathrm{n}(\%)$. ARB, angiotensin II receptor blocker; evolutional change, longitudinal assessment over a period of 1.5 years in patients on anti-hypertensive medication; regression change, longitudinal assessment from the start of anti-hypertensive medication to the end of 1 year after the start of the medication.

Other abbreviations as in Table 1.

Table 5. Longitudinal Change in baPWV and in BRS ${ }^{\dagger}$

\begin{tabular}{lcccc}
\multicolumn{1}{c}{$\begin{array}{c}\text { Significant } \\
\text { variable }\end{array}$} & $\begin{array}{c}\text { Non-standardization } \\
\text { coefficient (SE) }\end{array}$ & $\begin{array}{c}\text { Standardization } \\
\text { coefficient }\end{array}$ & t-value & P-value \\
$\begin{array}{c}\text { Total } \mathbf{R}^{2}=\mathbf{0 . 1 2 4} \\
\delta \text { baPWV } \ddagger\end{array}$ & $-0.020(0.008)$ & -0.467 & -2.453 & 0.020 \\
$\begin{array}{c}\text { Total } \mathbf{R}^{2}=\mathbf{0 . 0 9 1} \\
\delta \text { baPWV }\end{array}$ & $-0.005(0.002)$ & -0.357 & -2.997 & 0.004 \\
\hline
\end{tabular}

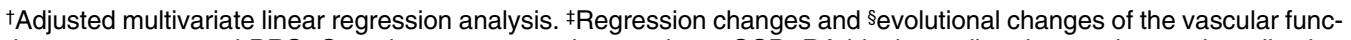
tion parameters and BRS. Covariates: age, gender, smokers, CCB, RA blockers, diuretics, statins, oral medication for DM, $\delta \mathrm{SBP}$, and $\delta \mathrm{HR}$. $\delta$, value at the end of the follow-up period minus that at baseline.

Other abbreviations as in Table 1.

baPWV, SP2, and BRS, but not rAI and FMD, showed significant improvement after anti-hypertensive medication for 1 year (Table 4). On univariate linear regression analysis only the change of baPWV from the start to the end of 1 year after the start of anti-hypertensive medication was significantly related to that of BRS (standardization coefficient, $-0.373 ; \mathrm{P}=0.018$ ). Multivariate linear regression analysis with the adjustment (change of SBP, that of heart rate, and anti-hypertensive medication were included as covariates) showed that change of baPWV during the follow-up period had a significant independent association with that of the BRS during the same period (Table 5).

Evolutional Change of Vascular Function Parameters and BRS Among the 218 subjects who were on anti-hypertensive medication, 140 agreed to be followed up at the outpatient de- partment of Tokyo Medical University Hospital, and vascular function tests and assessment of BRS were conducted successfully after 1.5 years after the initial vascular function tests and assessment of BRS in 107 subjects (15 subjects were excluded because $\beta$-blocker was prescribed during the follow-up period). During this period, baPWV, FMD and BRS showed no significant changes (Table 4). Heart rate, however, decreased significantly, and rAI and SP2 increased significantly (Table 4). On univariate linear regression analysis only change in baPWV during the follow-up period had a significant association with that of BRS during the same period (standardization coefficient, $-0.375 ; \mathrm{P}<0.001)$. On multivariate linear regression analysis with the adjustment (change of SBP, that of heart rate, and antihypertensive medication were included as covariates), change of baPWV during the follow-up period had a significant inde- 
pendent association with that of the BRS during the same period (Table 5).

\section{Discussion}

To the best of our knowledge, the present study is the first to confirm by not only cross-sectional assessment, but also longitudinal assessments, that baPWV, rather than AI/CBP or FMD, had a significant association with BRS in subjects with hypertension.

Several studies have demonstrated that arterial stiffness, as reflected by PWV, is associated with BRS. ${ }^{12} 16$ These findings, however, were derived from only cross-sectional studies. Only one prospective study has examined the association between change of arterial stiffness and that of BRS over time, and reported that renal transplantation improves systemic arterial stiffness accompanied by improvement of BRS; although AI, but not carotid-femoral PWV, also improved following renal transplantation in that prospective study. ${ }^{27}$ Thus, the strength of the present study is that it allowed confirmation of the association of baPWV with BRS on longitudinal assessments. It was found that change in BRS during a 1-year period after the start of antihypertensive medication had a significant association with that of baPWV during the same period; no such association of change in BRS with time was observed with that of AI/CBP or FMD. Furthermore, the evolutional change of BRS during a follow-up period $>1.5$ years in patients on anti-hypertensive medication had a significant association with that of baPWV, but not with that of AI/CBP or FMD. The present findings suggest that increase in stiffness of the large- to middle- sized arteries contributes to abnormal regulation of the baroreflex.

The present finding, that is, the existence of a significant association between PWV and BRS, may have 2 clinical implications, as follows. First, increased arterial stiffness is a risk factor for future cardiovascular events in subjects with hypertension. ${ }^{3}$ Increased cardiac afterload, impaired coronary blood flow supply, and/or microvascular damage in peripheral organs may be mechanisms underlying this association., ${ }^{1,2}$ Several studies have reported that abnormal cardiovascular autonomic nervous function is a risk factor for future cardiovascular events..$^{28,29}$ Thus, the present results suggest that abnormal autonomic nervous function is one of the mechanisms underlying PWVrelated increased risk of cardiovascular disease. And second, in the present study, the evolutional change and regression of baPWV were related to change in BRS over the same period of time. Improvement of abnormal autonomic nervous function has been reported to be beneficial for the reduction of blood pressure and improvement of hypertension-related disorders. ${ }^{6}$ Therefore, reduction of arterial stiffness may be an approach to improve abnormal autonomic nervous function.

$\mathrm{AI} / \mathrm{CBP}$ and FMD, other markers of vascular function, have been reported as independent markers of future cardiovascular events in subjects with hypertension. ${ }^{4,5}$ Increased AI is caused by abnormal central hemodynamics, such as elevation of CBP and increase of the central pulse pressure. ${ }^{1,4}$ Elevation of CBP acts to decrease BRS, ${ }^{9,10}$ whereas increase of the central pulse pressure may act to increase BRS via increased pulsatility. ${ }^{30}$ In contrast, FMD reflects endothelial NO bioavailability, and increased arterial elasticity derived from increased endothelial NO bioavailability may increase BRS. ${ }^{18}$ Chapleau et al reported that NO itself directly decreases baroreceptor activity, ${ }^{31}$ whereas others reported that NO production in the brain increases BRS. ${ }^{17}$ In addition, prostacyclin increases baroreceptor activity and oxygen free radicals decrease baroreceptor activity, ${ }^{19,20}$ both being substances related to endothelial function. Thus, the associations of AI/CBP and FMD with BRS are complex, and in the present study no significant association of BRS was observed with AI/CBP or FMD. Therefore, the contribution of abnormal autonomic nervous function to increased risk for cardiovascular disease related to abnormal central hemodynamics or impaired FMD may be small or not significant.

\section{Study Limitations}

BRS was determined by radial tonometry under spontaneous breathing. Although the limitations of this method have been noted, ${ }^{32}$ the entire spectrum of baroreflex stimulus-response was not investigated in the present study. baPWV reflects stiffness of the large- to middle-sized arteries, therefore, confirmation of the association of BRS with stiffness of larger arteries as reflected by carotid-femoral PWV is proposed. ${ }^{23,33}$ All of the present subjects were patients with hypertension, and we did not examine the significance of the association of BRS with vascular function in subjects with other conditions such as normal blood pressure or diabetes mellitus.

In the assessment of evolutional change, BRS and heart rate were significantly decreased and rAI and SP2 were significantly increased. Although the precise reasons why these parameters had significant changes during the follow-up period could not be clarified, aging and change of lifestyle such as habitual physical activity might affect these parameters. ${ }^{34,35}$ Some studies have reported that improvement of FMD is observed at 3-6 months after the start of anti-hypertensive medication. ${ }^{36,37}$ In the present study, however, no significant improvement of FMD was observed even at 1 year after the start of anti-hypertensive medication. One plausible explanation for this lack of improvement of FMD might be the difference in the time to re-examination of FMD after the start of anti-hypertensive medication, because aging reduces FMD. ${ }^{38}$

\section{Conclusions}

In addition to the cross-sectional assessment, the longitudinal assessments in the present study have shown that evolutional change and regression of baPWV, but not those of AI and FMD, had significant associations with change in BRS. Thus, increased stiffness of the large- to middle-sized arteries, rather than central hemodynamics or endothelial function, may contribute to abnormal baroreflex regulation.

\section{Conflict of interest: None to declare.}

Disclosures

\section{References}

1. Tomiyama H, Yamashina A. Non-invasive vascular function tests: Their pathophysiological background and clinical application. Circ J 2010; 74: 24-33.

2. Laurent S. Defining vascular aging and cardiovascular risk. J Hypertens 2012; 30(Suppl): S3-S8.

3. Munakata M, Konno S, Miura Y, Yoshinaga K; J-TOPP Study Group. Prognostic significance of the brachial-ankle pulse wave velocity in patients with essential hypertension: Final results of the J-TOPP study. Hypertens Res 2012; 35: 839-842.

4. Sung SH, Cheng HM, Wang KL, Yu WC, Chuang SY, Ting CT, et al. White coat hypertension is more risky than prehypertension: Important role of arterial wave reflections. Hypertension 2013; 61: 13461353.

5. Modena MG, Bonetti L, Coppi F, Bursi F, Rossi R. Prognostic role of reversible endothelial dysfunction in hypertensive postmenopausal women. J Am Coll Cardiol 2002; 40: 505-510.

6. Schlaich MP, Hering D, Sobotka PA, Krum H, Esler MD. Renal denervation in human hypertension: Mechanisms, current findings, and future prospects. Curr Hypertens Rep 2012; 14: 247-253.

7. Shimizu S, Akiyama T, Kawada T, Sata Y, Mizuno M, Kamiya A, 
et al. Medetomidine, an $\alpha_{2}$-adrenergic agonist, activates cardiac vagal nerve through modulation of baroreflex control. Circ J 2012; 76: 152_ 159.

8. Murozono Y, Yufu K, Takahashi N, Okada N, Shinohara T, Nakagawa $\mathrm{M}$, et al. Combined assessment of baroreflex sensitivity with iodine 123 metaiodobenzylguanidine scintigraphic findings strengthens the power of predictive value for cerebral and cardiovascular events in type 2 diabetic patients. Circ J 2013; 77: 130-136.

9. Abboud FM. The sympathetic system in hypertension: State-of-theart review. Hypertension 1982; 4(3 Pt 2): 208-225.

10. Lanfranchi PA, Somers VK. Arterial baroreflex function and cardiovascular variability: Interactions and implications. Am J Physiol Regul Integr Comp Physiol 2002; 283: R815-R826.

11. Hunt BE, Fahy L, Farquhar WB, Taylor JA. Quantification of mechanical and neural components of vagal baroreflex in humans. $H y-$ pertension 2001; 37: 1362-1368.

12. Okada Y, Galbreath MM, Shibata S, Jarvis SS, VanGundy TB, Meier RL, et al. Relationship between sympathetic baroreflex sensitivity and arterial stiffness in elderly men and women. Hypertension 2012; 59: 98-104.

13. Monahan KD, Tanaka H, Dinenno FA, Seals DR. Central arterial compliance is associated with age- and habitual exercise-related differences in cardiovagal baroreflex sensitivity. Circulation 2001; 104: $1627-1632$

14. Mattace-Raso FU, van den Meiracker AH, Bos WJ, van der Cammen TJ, Westerhof BE, Elias-Smale S, et al. Arterial stiffness, cardiovagal baroreflex sensitivity and postural blood pressure changes in older adults: The Rotterdam Study. J Hypertens 2007; 25: 1421-1426.

15. Michas F, Manios E, Stamatelopoulos K, Koroboki E, Toumanidis $\mathrm{S}$, Panerai RB, et al. Baroreceptor reflex sensitivity is associated with arterial stiffness in a population of normotensive and hypertensive patients. Blood Press Monit 2012; 17: 155-159.

16. Radaelli A, Castiglioni P, Balestri G, Cesana F, De Carlini C, Soriano $\mathrm{F}$, et al. Increased pulse wave velocity and not reduced ejection fraction is associated with impaired baroreflex control of heart rate in congestive heart failure. J Hypertens 2010; 28: 1908-1912.

17. Campos RR, de Camargo AC, Ulrich H. Brain nitric oxide production by a proline-rich decapeptide from Bothrops jararaca venom improves baroreflex sensitivity of spontaneously hypertensive rats. Hypertens Res 2010; 33: 1283-1288.

18. Sudano I, Roas S, Noll G. Vascular abnormalities in essential hypertension. Curr Pharm Des 2011; 17: 3039-3044.

19. Chen HI, Chapleau MW, McDowell TS, Abboud FM. Prostaglandins contribute to activation of baroreceptors in rabbits. Possible paracrine influence of endothelium. Circ Res 1990; 67: 1394-1404.

20. Li Z, Mao HZ, Abboud FM, Chapleau MW. Oxygen-derived free radicals contribute to baroreceptor dysfunction in atherosclerotic rabbits. Circ Res 1996; 79: 802-811.

21. Tomiyama H, Kohro T, Higashi Y, Takase B, Suzuki T, Ishizu T, et al. A multicenter study design to assess the clinical usefulness of semiautomatic measurement of flow-mediated vasodilatation of the brachial artery. Int Heart J 2012; 53: 170-175.

22. Tomiyama H, Yamazaki M, Sagawa Y, Teraoka K, Shirota T, Miyawaki Y, et al. Synergistic effect of smoking and blood pressure on augmentation index in men, but not in women. Hypertens Res 2009; 32: $122-126$.

23. Yamashina A, Tomiyama H, Takeda K, Tsuda H, Arai T, Hirose K, et al. Validity, reproducibility, and clinical significance of noninvasive brachial-ankle pulse wave velocity measurement. Hypertens Res 2002; 25: $359-364$.

24. Tomiyama H, Matsumoto C, Yamada J, Teramoto T, Abe K, Ohta H, et al. The relationships of cardiovascular disease risk factors to flowmediated dilatation in Japanese subjects free of cardiovascular disease. Hypertens Res 2008; 31: 2019-2025.

25. Robbe HW, Mulder LJ, Rüddel H, Langewitz WA, Veldman JB, Mulder G. Assessment of baroreceptor reflex sensitivity by means of spectral analysis. Hypertension 1987; 10: 538-543.

26. Jones A, Beda A, Ward AM, Osmond C, Phillips DI, Moore VM, et al. Size at birth and autonomic function during psychological stress. Hypertension 2007; 49: 548-555.

27. Kaur M, Chandran D, Lal C, Bhowmik D, Jaryal AK, Deepak KK, et al. Renal transplantation normalizes baroreflex sensitivity through improvement in central arterial stiffness. Nephrol Dial Transplant 2013; 28: 2645-2655

28. Ko SH, Song KH, Park SA, Kim SR, Cha BY, Son HY, et al. Cardiovascular autonomic dysfunction predicts acute ischaemic stroke in patients with type 2 diabetes mellitus: A 7-year follow-up study. Diabet Med 2008; 25: 1171-1177.

29. Barron HV, Lesh MD. Autonomic nervous system and sudden cardiac death. J Am Coll Cardiol 1996; 27: 1053-1060.

30. Chapleau MW, Abboud FM. Determinants of sensitization of carotid baroreceptors by pulsatile pressure in dogs. Circ Res 1989; 65: $566-$ 577.

31. Chapleau MW, Hajduczok G, Sharma RV, Wachtel RE, Cunningham JT, Sullivan MJ, et al. Mechanisms of baroreceptor activation. Clin Exp Hypertens 1995; 17: 1-13.

32. Smith SM, Potter JF, Samani NJ, Sammons EL, Rathbone WE, Bentley S, et al. Are baroreflex events detected by invasive and noninvasive techniques coincident? Clin Physiol Funct Imaging 2008; 28: $262-269$.

33. Laurent $S$, Boutouyrie P. Arterial stiffness: A new surrogate end point for cardiovascular disease? J Nephrol 2007; 20 (Suppl 12): S45-S50.

34. Fisher JP, Kim A, Hartwich D, Fadel PJ. New insights into the effects of age and sex on arterial baroreflex function at rest and during dynamic exercise in humans. Auton Neurosci 2012; 172: 13-22.

35. Jones WS, Vemulapalli S, Patel MR. Interventional treatment of hypertension: A new paradigm. Curr Cardiol Rep 2013; 15: 356.

36. Li M, Xu A, Lam KS, Cheung BM, Tse HF. Impact of combination therapy with amlodipine and atorvastatin on plasma adiponectin levels in hypertensive patients with coronary artery disease: Combination therapy and adiponectin. Postgrad Med 2011; 123: 66-71.

37. Ghiadoni L, Magagna A, Kardasz I, Taddei S, Salvetti A. Fixed dose combination of perindopril and indapamide improves peripheral vascular function in essential hypertensive patients. Am J Hypertens 2009; 22: 506-512.

38. Seals DR, Jablonski KL, Donato AJ. Aging and vascular endothelial function in humans. Clin Sci (Lond) 2011; 120: 357-375. 